\title{
Erratum to: PKM2 and ACVR 1C are prognostic markers for poor prognosis of gallbladder cancer
}

\author{
J. Li $\cdot$ Z. Yang $\cdot$ Q. Zou $\cdot$ Y. Yuan $\cdot$ \\ J. Li $\cdot$ L. Liang $\cdot$ G. Zeng $\cdot$ S. Chen
}

Published online: 26 October 2013

(c) Federación de Sociedades Españolas de Oncología (FESEO) 2013

\section{Erratum to: Clin Transl Oncol \\ DOI 10.1007/s12094-013-1063-8}

In the published original article, some details in the affiliation of the first author J. Li are missing. The correct affiliation is given below.

\section{J. Li}

Department of General Surgery, Second Xiangya Hospital, Central South University, Changsha 410011, Hunan, People's Republic of China

The online version of the original article can be found under doi:10.1007/s12094-013-1063-8.

\section{J. Li}

Department of General Surgery, Second Xiangya Hospital, Central South University, Changsha 410011, Hunan, People's Republic of China

\section{Z. Yang $(\bowtie)$}

Research Laboratory of Hepatobiliary Diseases, Second Xiangya Hospital, Central South University, Changsha 410011, Hunan, People's Republic of China e-mail: zhulinyang@yahoo.com

\section{Q. Zou · Y. Yuan}

Department of Pathology, Third Xiangya Hospital, Central South University, Changsha 410011, Hunan, People's Republic of China

J. Li

Department of Pathology, Xiangya Medical School, Central South University, Changsha 410011, Hunan, People's Republic of China

\author{
L. Liang \\ Department of Hepatobiliary and Pancreatic Surgery, \\ Hunan Provincial People's Hospital, Changsha 410007, \\ Hunan, People's Republic of China

\section{G. Zeng} \\ Department of Pathology, Loudi Central Hospital, \\ Loudi 417011, Hunan, People's Republic of China \\ S. Chen \\ Department of Pathology, Hunan Provincial Tumor Hospital, \\ Changsha 410013, Hunan, People's Republic of China
}

